\title{
VIDEO RETRIEVAL AND RELEVANCE FEEDBACK IN THE CONTEXT OF A POST-INTEGRATION MODEL
}

\author{
Roy Wang, Milind R. Naphade, and Thomas S. Huang \\ Department of Electrical and Computer Engineering \\ Beckman Institute for Advanced Science and Technology \\ University of Illinois at Urbana-Champaign \\ \{milind,rwang,huang\}@ifp.uiuc.edu
}

\begin{abstract}
Video can be viewed as the integration of several heterogeneous media interwoven in a temporally close-coupled fashion. To support the retrieval of video segments under the query-by-example scenario, we purport in this paper a post-integration model that integrates low-level media types to identify visually/auditorily similar video segments. The model also allows flexible relevance feedback on the user end to further improve the speed and accuracy of searching in a video database. As its name implies, the post-integration model first treats models of the underlying media as independent processes and then combines distance scores from each of the underlying media at the later stage. This decoupling allows the usage of efficient algorithms to fast and accurately compare thousands of video clips in a moderate-to-large-sized video database. It also naturally facilitates improved user interaction by fast dynamic weight adjustment on the media distance scores in a multiple query succession. We explicit our models and dynamic weight adjustment schemes in this paper and offer demonstration with a working system on a large video database set.
\end{abstract}

\section{INTRODUCTION}

Recently we have witnessed a great deal of enthusiasm and emergence of wide varieties of tools to model, represent, search image and video database. As it has been demonstrated by numerous image search engines and retrieval systems [1], we would very much like to have a video retrieval system that have similar performance level. In this paper, we propose a post-integration model to address the query-by-example scenario in the video search and retrieval field. We decouple the treatment of individual media comparison and media combination to allow efficient and accurate temporal pattern matching and parameter estimation. Some successful techniques such as relevance feedback used in image retrieval will be introduced in the video domain in the context of this model.

At the basis of our ascent to ultimate semantic-level video retrieval, we have at our disposal a great number of tools for parsing low-level video contents, and sometimes even high-level video structures in specific domains. Shot segmentation, representation by key frames, scene construction with or without domain restrictions have all been proposed and analyzed in sufficiently large number of papers in the past. With 
these tools, we would thus aim to build a video retrieval system to support query-byexample video clips and ultimately query-by-keywords. The latter is difficult in the sense that each key-word is an inherent multimodal pattern recognition problem. Quite a number of existing systems support query-by-content, in some cases, sketchs/icons supplied as queries, such as but limited to the following: [2], [3], [4],[5],[6], [7] etc.

Query-by-example (QBE) systems lie in-between low-level and high-level processing arenas in terms of representation power and performances. It carries enough representation power for us to expect it to provide visually/auditorily similar query results. In the QBE paradigm, two tasks are dominant. The first is to produce a compact signature representation of video segments(normally a segment is one camera shot). The second is to provide algorithms to compare different signatures from different segments. In our QBE system, we address the important issue of similarity metric of temporal patterns and the integration of different media thereafter. We cast the problem of video segment matching into a dynamic programming framework. To further enhance retrieval results, we perform relevance feedback upon user selection of desired and undesired return segments. In image counterparts, relevance feedback adjust weights corresponding to feature units (e.g. weight on color feature) and subunits (e.g. weight on specific color histogram bins). The important distinction of the post-integration model is that the weights are applied to the distance scores resulting from pairwise comparison results. This difference is due to the fact that we avoid computing a direct compact signature for each media. We circumvented the difficult modelling problem by transferring the burden to model to the burden to compare. This eases the modelling of video content; yet creates difficulties in incorporating user feedbacks, and carries some computational side-effects. In our implemented system, we have incorporated four types of media: color(expressed in terms of histograms), audio(expressed in MFC coefficients),edge(directional histogram), motion(overall motion histogram). The system does not prohibit incorporation of additional features or individual feature representation.

In followings sections, we first briefly illustrate the dynamic programming approach as the tool for measuring distances in a single media type. Then we propose relevance feedback to allow dynamic combination of different media in the context of our post-integration model. Furthermore, we point out several implementation issues in weighted combination, positive/negative feedbacks and propose solutions to the problems. Lastly, we demonstrate retrieval results and relevance feedback results in a large video database setting, draw conclusion and discuss future enhancements.

\section{SIMILARITY METRIC BETWEEN VIDEO SEGMENTS}

We cast the problem of defining distance between two temporally patterns with varying lengths as a dynamic programming problem. Let $\mathcal{X}=\left\{\vec{x}_{1}, \ldots, \vec{x}_{T_{X}}\right\}$ and $\mathcal{Y}=\left\{\vec{y}_{1}, \ldots, \vec{y}_{T_{Y}}\right\}$ be the sequence of feature vectors (of a single feature stream) of two video clips, which need to be matched. To match the two signatures $\mathcal{X}$ and $\mathcal{Y}$ with non-identical time periods $T_{X}$ and $T_{Y}$, we need to align the beginning and end of the sequences and normalize the two temporally. To define dissimilarity in a more 
general way, we define two warping functions, which relate to a common time axis and warp the two patterns to this common normal time axis resulting in a nonlinear time normalization. Defining two warping functions $\Phi_{X}$ and $\Phi_{Y}$ such that

$$
i_{x}=\Phi_{X}(k) ; k=1, \ldots, T
$$

and

$$
i_{y}=\Phi_{Y}(k) ; k=1, \ldots, T
$$

Defining a general dissimilarity measure based on the warping functions in Equations 1,2 as

$$
d_{\Phi}(\mathcal{X}, \mathcal{Y})=\sum_{k=1}^{T} d\left(\Phi_{X}(k), \Phi_{Y}(k)\right) m(k) / M(\Phi)
$$

where $d\left(\Phi_{X}(k), \Phi_{Y}(k)\right)$ is the short time distortion defined for $x_{\Phi_{X}(k)}$ and $y_{\Phi_{Y}(k)}$, $m(k)$ is a non-negative path weighting coefficient and $M_{\Phi}$ is a path normalizing factor. Since there is an extremely large number of possible warping function pairs $\Phi=$ $\left(\Phi_{X}, \Phi_{Y}\right)$, we need a rule to select one of these function pairs. One way to is to define the dissimilarity $d(\mathcal{X}, \mathcal{Y})$ as the minimum of $d_{\Phi}(\mathcal{X}, \mathcal{Y})$, over all possible paths, such that

$$
d(\mathcal{X}, \mathcal{Y})=\min _{\Phi} d_{\Phi}(\mathcal{X}, \mathcal{Y})
$$

where $\Phi$ is subject to certain constraints that involve local warping and global warping direction and range.

This is equivalent to finding the best warping function pair i.e. the best path through a grid mapping the features of one pattern to those of the other. Dynamic time warping is the natural choice for this optimization. Overall distance across different media types is expressed as the weighted sum of individual media. For further details on the algorithm, please refer to [8].

\section{RELEVANCE FEEDBACK AND COMBINATION OF MEDIA}

In previous section, we have constructed the distance metric between video units. On top of this defined distance measure between shots, we propose a optimal (in square norm sense) linear weight updating method to incorporate user feedbacks to achieve more accurate retrievals. Relevance feedback has been used in the past for image retrieval [9]. The purpose of feedback is to allow faster pinpointing the desired targets of the user's query.

As mentioned in the introduction, we formulate our feedback as the weight updating over individual media distances of pairwise comparison. To keep the model simple, we assume that the overall video unit distance is a linear combination of different media distances. The relevance feedback target thus involves computing the solution for the best weighted linear transform of the feature distances in ranking the desired returns on top of others.

Let us now assume that the user selects $M$ video clips, retrieved during the previous iteration of query as relevant returns. Let us further assume that the user sets 
the relevance of each of these clips on a scale between 0 and 1 , with larger values denoting more relevance. Let us assume that $M>N$, where $N$ is the number of feature streams. We now need to compute the optimal set of feature stream weights $w_{j}, j \in\{1, \ldots, N\}$. Let $D$ be the distortion matrix, where $D=\left\{d_{i j}\right\}$, $j \in\{1, \ldots, N\}, i \in\{1, \ldots, M\}$. The distortion vector for each selected clip $\vec{d}_{i}$ is then multiplied by the relevance of that clip $r_{i}$ to obtain the matrix $R$.

$$
R^{T}=\left[r_{1} * \vec{d}_{1}^{T}|\ldots| r_{M} * \vec{d}_{M}^{T}\right]
$$

The overall distortion vector for the $M$ clips is given by $D \cdot \vec{w}$. In order to compute the optimal weight vector based on the user's relevance feedback, we then minimize the function in Equation 5

$$
C(\vec{w})=\vec{w}^{T} R^{T} R \vec{w}
$$

The $N \times N$ matrix $R^{T} R$ is positive semi-definite. If we assume that at least $N$ of the vectors $\vec{d}_{i}$ are linearly independent, then $R^{T} R$ is positive definite. In this case, the unit norm solution (to avoid degeneracy at zero vector), the desired weight vector $w$ becomes the eigenvector corresponding to the smallest eigenvalue of matrix $R^{T} R$. Let $\left\{\lambda_{i}\right\}$ and $\left\{e_{i}\right\}$ be the eigenvalues and vectors associated with matrix $R^{T} R$ and $\lambda_{1}>\lambda_{2} . .>\lambda_{N}$. Any weight vector $\vec{w}$ can be decomposed as $u_{1} \overrightarrow{e_{1}}+. .+u_{N} \overrightarrow{e_{N}}$, where $u_{1}^{2}+. . u_{N}^{2}=1$. Setting the optimal $\overrightarrow{w^{*}}=\vec{e}_{1}$ and assuming without loss of generality that $\left\|\overrightarrow{e_{i}}\right\|=1$

$$
\begin{aligned}
C(\vec{w})-C\left(\vec{w}^{*}\right)= & \left.\vec{w}^{T}\left(R^{T} R\right) \vec{w}-\vec{e}_{1}^{T}\left(R^{T} R\right) \vec{e}_{1}\right) \\
& =\lambda_{1} u_{1}^{2}+. .+\lambda_{N} u_{N}^{2}-\lambda_{1} \\
& \geq \lambda_{1}\left(u_{1}^{2}+\ldots u_{N}^{2}-1\right)=0
\end{aligned}
$$

Several issues complicate the treatment of the solution of equation 5. 1) There exists the possibility of one or several weight vector elements to be negative. This does not reflect the intuition that one media should be discarded not included from computation when it's found to be ineffective. 2)Negative user relevance feedback are not treated differently than positive feedbacks. The simple mixing of the two can produce weighting to bias towards meaningless solution. 3) User can return much fewer images than needed to solve the equations. This third issue can be dealt with by simply accumulating user returns until the rank of matrix $R$ is sufficient to perform eigen decomposition over the covariance matrix $R^{T} R$.

Issue 1) is nothing more than additional constraints in our optimization problem. The additional inequality constraints are $w_{1}>0, . ., w_{N}>0$. The combination of the original second order non-linear equality constraint with the added linear inequality constraints makes optimization difficult. We realize that the total constraints describe a convex set of hemi-spherical surface that the optimal solutions can lie in. We propose a simple local optimal solution by gradient descent over the the convex solution. The starting point is the optimal vector found without the additional inequality constraints. We then fix all negative elements to zero, and start gradient descents from that point on. The gradient of the quadratic cost function is simply given as $2 R \vec{w}$. With $\mu$ being 


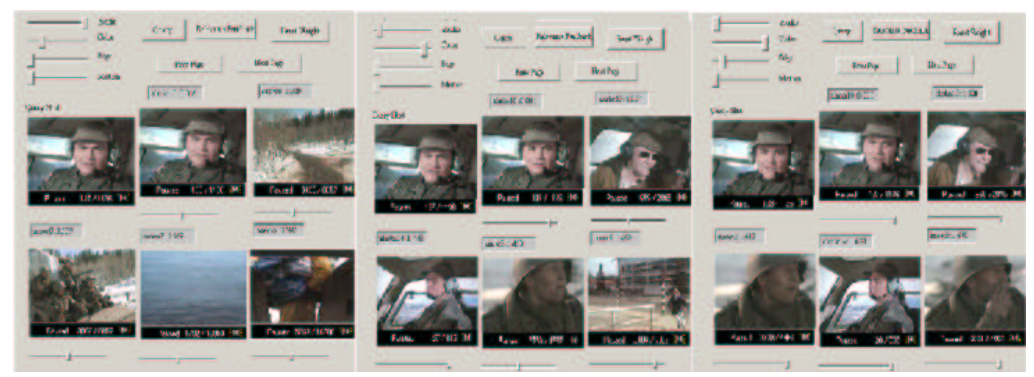

Figure 1: from left to right: initial return, 1st and 2nd user relevance feedback returns. see text

the step size, We have

$$
w_{n e w}=\frac{w_{\text {old }}+\mu * 2 R \vec{w}}{\left\|w_{o} l d+\mu * 2 R \vec{w}\right\|}
$$

This creates the minimal projection distance to the convex set after the gradient walk with $\mathrm{c}$ as the step size. This approach might not produce a globally optimizer, however;

Issue 2 prompts us to separate the treatment of positive feedbacks and negative feedbacks. The positive feedbacks give us the definitive evidence of where we would like the weight to go. The negative feedbacks are sparse samples of a larger class of un-wanted query returns and the estimated weight negation should be treated with lower confidence than the positive feedback changes. In practice, we found that stable results are achieved by adding the solution of negative feedback to the solution of the positive feedback with a small weight. $w_{\text {new }}=w_{\text {pos }}+\alpha * w_{\text {neg }}$ where $\alpha<0.5$.

\section{RESULTS}

We have implemented the proposed retrieval framework over a large database comprising of several thousands of video shots from dozens of cinematic movies. Due to space constraint, we only show query runs on one video query. 1. The leftmost is the initial system returns with initial random weighting(top-left being the query shot itself). The right two show the two consecutive user feedback return results. The slide bars on the top-left portion of each screenshot show the weights related to audio, color, edge, and motion feature streams. At the end of 2 nd round, the color weight is increased to reflect the user's desired direction and the top query returns are satisfactory in terms of visual and auditory similarity.

\section{CONCLUSION}

In this paper we report a post-integration model treatment to the query-by-example problem in video retrieval. The model decouples the matching of individual media and 
the relevance feedback combination of individual media distances. Our system implemented over a large video data set produces visually/auditorially satisfactory returns due to the unique ability of combining temporal pattern matching and media integration. We also point out the limitation of the approach, specifically and inevitably from the fact that it is inherently a linear integration over media types. We are investigating models that can dynamically affect the time warping stage thus allowing more flexible, potentially non-linear treatment of media combination. We are further looking at improving individual media's feature representation along with adding medium and high level semantic concept representation in the retrieval system.

\section{References}

[1] A. Smeulders, M. Worring, S. Santini, A. Gupta, and R. Jain, "Content-based image retrieval at the end of the early years," PAMI, vol. 22, pp. 1349-1380, December 2000.

[2] M. Flickner, H. Sawhney, W. Niblack, J. Ashley, Q. Huang, B. Dom, M. Gorkani, J. Hafner, D. Lee, D. Petkovic, D. Steele, and P. Yanker, "Query by image and video content: The QBIC system,” IEEE Computer, vol. 28, no. 9, pp. 23-32, 1995.

[3] J. Bach, C. Fuller, A. Gupta, A. Hampapur, B. Horowitz, R. Humphrey, R. Jain, and C. Shu, "The virage image search engine: An open framework for image management," in Proceedings of SPIE Storage and Retrieval for Image and Video Databases, Feb. 1996.

[4] J. R. Smith and S. F. Chang, "Visualseek: A fully automated content-based image query system," in Proceedings of ACM Multimedia, (Boston, MA), Nov. 1996.

[5] S. Srinivasan, D. Ponceleon, A. Amir, and D. Petkovic, "What is that video anyway? In search of better browsing," in Proceedings of IEEE International Conference on Multimedia and Expo, pp. 388-392, July 2000.

[6] W. Ma and B. S. Manjunath, "NETRA: A toolbox for navigating large image databases," Multimedia Systems, vol. 7, no. 3, pp. 184-198, 1999.

[7] M. M. Yeung and B. Liu, "Efficient matching and clustering of video shots," in Proceedings of IEEE International Conference on Image Processing, vol. 1, (Washington, D.C.), pp. 338-341, Oct. 1995.

[8] M. R. Naphade, R. Wang, and T. S. Huang, "Multimodal pattern matching for audio-visual query and retrieval," in Proceedings of SPIE, Storage and Retrieval for Media Databases, vol. 4315, pp. 188-195, Jan. 2001.

[9] Y. Rui, T. Huang, M. Ortega, and S. Mehrotra, "Relevance feedback: A power tool in interactive content-based image retrieval," IEEE Transactions on Circuits and Systems for Video Technology, Special Issue on Segmentation, Description and Retrieval of Video Content, vol. 8, pp. 644-655, Sep. 1998. 